\title{
Research progress of natural compounds in anti-liver fibrosis by affecting autophagy of hepatic stellate cells
}

\author{
Yongxiang Shu ${ }^{1} \cdot$ Xuyou Liu $^{1} \cdot$ Haifeng Huang ${ }^{1} \cdot$ Qi Wen $^{1} \cdot$ Jianchang Shu ${ }^{1}(\mathbb{C}$
}

Received: 2 June 2020 / Accepted: 19 January 2021 / Published online: 20 February 2021

(C) The Author(s) 2021

\begin{abstract}
Chronic liver diseases caused by various pathogenesis are marked by inflammatory infiltration and wound healing reaction, while their normal regeneration ability is impaired. The unbalance between the generation and the degradation of extracellular matrix (ECM) leads to collagen accumulation and develops into liver fibrosis. Inflammation, oxidative stress, and autophagy interact closely in the pathogenesis of hepatic fibrosis. Reactive Oxygen Species (ROS) can not only stimulate Kupffer cells to release massive inflammatory factors, but induce autophagy. However, the latter may suppress inflammatory reaction by inhibiting proinflammatory complex formation directly, and removing damaged organelles or pathogenic microorganism indirectly. At present, effective anti-fibrosis drugs are still lacking. Previous studies have found various natural compounds enabled liver protection through anti-inflammatory, antioxidant, and other mechanisms. In recent years, autophagy, a vital life activity, has been found to be involved in the mechanism of liver fibrosis. As a new target, developing anti-liver fibrosis drugs that regulate the activity of autophagy is very promising. In this review, we summarize the latest studies about natural compounds in the treatment of liver fibrosis by regulating autophagy.
\end{abstract}

Keywords Liver fibrosis $\cdot$ Hepatic stellate cell $\cdot$ Natural compound autophagy $\cdot$ Apoptosis $\cdot$ Senescence

\section{Introduction}

Chronic liver diseases caused by various causes is characterized by inflammatory infiltration and wound healing response, but the normal regeneration of liver cells is impaired. Finally, the imbalance between the generation and degradation of extracellular matrix leads to the accumulation of collagen and liver fibrosis (LF) [1]. Inflammation, oxidative stress and autophagy are closely related in the pathogenesis of LF. Unfortunately, due to the shortage of

Jianchang Shu

shujc0328@163.com

Yongxiang Shu

849247207@qq.com

Xuyou Liu

Iiuxy717@163.com

Haifeng Huang

1143644182@qq.com

Qi Wen

243136784@qq.com

1 Department of Gastroenterology, GuangZhou Red Cross Hospital, Jinan University, Guangzhou 510220, China effective clinical treatment strategies [2, 3], exploring new therapeutic targets and drugs to reverse liver fibrosis remains an inevitable and tough task.

Autophagy, the hot research spot in recent years, has been shown to be involved in the pathogenesis of a lot of human diseases, such as cardiovascular diseases, neurodegenerative diseases, and tumors. Microtubule-associated proteins 1 light chain 3B (LC3B), p62/sequestosome1 (SQSTM1) and beclin-1 are the three widely used autophagy markers. LC3B will be successively processed and modified into two forms: LC3-I and LC3-II when autophagy is initiated. Cytoplasmic LC3(LC3-I) will enzymatically dissolve a small segment of polypeptide and change to the autophagosome membrane type (LC3-II). The autophagy level is, thus, estimated by the ratio of LC3-II/LC3-I [4]. Beclin-1 is one of the most important autophagy regulating genes. It forms a complex with Class III PI3K, mediating the expression of other autophagy proteins. P62, also known as SQSTM1, is a ubiquitin-like binding protein. P62, as a regulatory factor, is conjured to LC3 and participates in the formation of autophagosome. There is a negative correlation between the overall expression level of p62 in cells and autophagy activity [5]. 
Currently, an increasing number of studies have noticed the influence of autophagy in the pathogenesis of liver fibrosis, and the research field of HSCs autophagy is drawing more interest.

It has been a long history that natural products were used by humans as medicine to treat diseases. It also has been an important part of modern medicine to extract active ingredients from natural products. A large number of natural compounds have advantageous properties such as safety and effectiveness, some of which are used as a main source of anti-fibrotic drugs.

\section{The pathophysiological mechanism and therapeutic progress of hepatic fibrosis}

The pathophysiological mechanism of liver fibrosis is very complex, involving a variety of cells and cytokines. Activation of HSCs is the central event of hepatic fibrosis, which has become the consensus [6]. HSCs activation is a pathological process characterized by a phenotypic transdifferentiation of HSCs to myofibroblastic-like cell. These "new" myofibroblasts exhibit the following characteristics: proliferation, chemotaxis, contractility, releasing inflammatory cytokines, loss of intracellular vitamin A droplets [7]. They synthesize various extracellular matrix components such as different types of collagens as well as proteins, all of which are implicated in the beginning of the fibrotic process. The mechanism of HSCs activation includes epithelial cell damage, changes in extracellular matrix, immune regulation, molecular imbalance mediated by membrane receptors and nuclear receptors, disorder of epigenetics transcription, cellular homeostasis and stress disorder which includes autophagy, and so on. These mentioned above are described in detail in a review published by Higashi $\mathrm{T}$ et al. [8]. In general, once HSCs become activated, they show "permanent" characteristics. However, it has been demonstrated that HSCs can return to a dormant state when the damage factors are removed, which may also explain the fact that liver fibrosis and cirrhosis can be reversed in some patients.

Although there have been a lot of studies on the pharmacotherapy of liver fibrosis, few of them have been used clinically, because the efficacy of these drugs is uncertain. At present, according to the mechanisms, treatment directions mainly include the following: (1) primary diseases therapy; (2) Using liver-protecting agent to reduce the damage; (3) Blocking myofibroblast activation, contraction and fibrosis formation; (4) Promoting apoptosis or reversal of activated stellate cells; (5) Stimulating matrix degradation [9].

\section{The role of autophagy in HSCs and LF}

Autophagy is a catabolism process in which eukaryotic cells maintain cellular stability and organelle integrity by degrading intracytoplasmic components under stress. A certain degree of autophagy is essential to maintain the physiological function and normal metabolism. However, excessive enhancement or attenuation can lead to cell metabolism imbalance, thus causing the occurrence of diseases [10]. Previous studies have confirmed autophagy as a "double-edged sword" in a lot of diseases. Similarly, several recent studies revealed that the pathological mechanisms of many liver diseases may be autophagy dependent, such as hepatitis, hepatic steatosis, fibrosis, cirrhosis, and hepatocellular carcinoma [11].

At the moment, most studies tend to agree that when HSCs are stimulated, the autophagy level increases and provides energy for HSCs activation by promoting lipid degradation [12]. Therefore, enhanced autophagy is an accelerant for HSCs activation and hepatic fibrosis and reducing it to inhibit HSCs activation may be a therapeutic target. Indeed, previous studies by our group have shown that curcumin can inhibit autophagy and activity of LX-2 (a kind of immortalized HSC cell line) in vitro, and reduce autophagy level of liver tissue in vivo as well as the degree of liver fibrosis induced by $\mathrm{CCl} 4$ in rats, and it is speculated that the effect of curcumin on liver fibrosis is related to the inhibition of aHSCs autophagy [13, 14]. This is consistent with the view above.

However, some studies reported that the role of autophagy on LF and HSCs was diametrically opposed. For example, Seo HY et al. [15] concluded that after treatment of HSCs with phospholipase D1(PLD1), the production of I-type collagen (collagen I) had been decreased due to promoting autophagy. When autophagy was inhibited, the effect of PLD1 on collagen I had lost. What's more, Liu, a member of our team, recently reported that overexpressing of microRNA-148a increased autophagic activity, which inhibited proliferation and promoted apoptosis in HSCs [16]. These illustrate that promoting autophagy may be a potential anti-fibrotic approach.

Although the certain reasons for these opposite conclusions are still unclear, some scholars believe that the different effects of autophagy on the fate of HSCs depend on the degree of autophagy, and the extent of autophagy depends on the intensity and duration of cellular stress. It is well known that either over-upregulation or down-regulation of autophagy is detrimental to cell survival. When quiescent HSCs are stimulated, autophagy promotes HSCs activation by altering lipid metabolism, and at this time, inhibition of autophagy may lead to decreased activity or apoptosis of aHSCs by interrupting energy supply. While, 
in activated HSCs (aHSCs), excessive autophagy can lead to HSCs senescence, autophagic cell death or apoptosis, collagen degradation and inflammation inhibition, alleviating liver fibrosis [17].

\section{Natural compounds exert anti-liver fibrosis effect via autophagy}

Natural compounds can exert anti-fibrotic effects via a variety of mechanisms that mediate the activation of HSCs. Since autophagy is a hot topic in life science, more and more researchers now focus on the role of autophagy in LF, trying to find new methods to treat it by intervening autophagy. Studies show that natural compounds can reduce LF through influencing the activity of HSCs by regulating inflammation, promoting senescence and autophagic cell death /apoptosis of HSCs. This article summarizes the newest studies to provide innovative ideas for scientific and clinical research (Table 1), and gives the molecular formulas and chemical structures of some of the natural compounds (Table 2).

\section{Natural compounds inhibiting HSCs activation by down-regulating autophagy}

Trolline is a kind of isoquinoline alkaloid isolated from the flowers of Trollius chinensis Bunge (Ranunculaceae). Bai [18] et al. found that trolline significantly reduced $\mathrm{CCl}_{4}$-induced SD rats' liver injury and collagen deposition. Additionally, They found that trolline inhibited HSCT6 autophagy by down-regulating the ratio of LC3-II/ LC3-I, Beclin-1 and p62 which were the major proteins of autophagy. What's more, Atg5 and Atg7, the two key genes involved in conversion LC3-I to LC3-II, also decreased in the level of transcription. Therefore, they speculated trolline may partially inhibit the activation of HSCs by downregulating autophagy. However, their studies lack a control group that includes autophagy agonist as well as related signaling pathways.

Shikonin is a kind of natural product extracted from the roots of Lithospermum erythrorhizon and has anti-inflammatory, anti-tumor and antioxidant characteristics [19]. Liu [20] et al. found that shikonin visibly alleviated $\mathrm{CCl} 4$ and BDL-induced LF in mice. In addition, shikonin obviously inhibited the activity of aHSCs and downregulated the level

Table 1 Effects of natural compounds on HSC autophagy and related mechanisms

\begin{tabular}{|c|c|c|c|c|}
\hline Natural compounds & Direction of autophagy & & Molecular mechanism & References \\
\hline Trolline & Autophagy down-regulation & & $\begin{array}{l}\text { LC3-II/LC3-I ratio, Beclin-1, } \\
\text { P62, Atg5, Atg7(-) } \rightarrow \text { autophagy (-) }\end{array}$ & {$[18]$} \\
\hline Shikonin & & & $\begin{array}{l}\text { TGF- } \beta 1 / \text { Smads }(-) \rightarrow \text { Beclin- } 1(-) \\
\rightarrow \text { autophagy }(-)\end{array}$ & {$[20]$} \\
\hline Taurine & & & $\operatorname{PPAR} \alpha(-) \rightarrow$ autophagy $(-)$ & {$[22]$} \\
\hline Dihydroartemisinin (DHA) & Autophagy up-regulation & Inhibiting inflammation & $\begin{array}{l}\text { ROS-JNK } \\
1 / 2(+) \rightarrow \text { autophagy }(+) \rightarrow \text { inflammation } \\
\text { reaction }(-)\end{array}$ & {$[24]$} \\
\hline Catalpol & & & $\begin{array}{l}\text { LC3-II 、Beclin-1(+) } \\
\text { P62、IL-6、IL-8、TNF-a(-) } \\
\rightarrow \text { autophagy }(+)\end{array}$ & {$[26]$} \\
\hline $\begin{array}{l}\text { Methyl Helicterate } \\
(\mathrm{MH})\end{array}$ & & Inducing HSCs apoptosis & $\begin{array}{l}\mathrm{JNK}(+) \\
\mathrm{PI} 3 \mathrm{~K} / \mathrm{Akt} / \mathrm{mTOR}(-) \\
\rightarrow \text { autophagy }(+)\end{array}$ & [29] \\
\hline Purple pitanga extract & & & $\begin{array}{l}\text { autophagy/mitochondrial autophagy } \\
(+) \rightarrow \text { apoptosis }(+)\end{array}$ & {$[30]$} \\
\hline Caffeine & & & $\begin{array}{l}\text { IER } 1-\mathrm{a}(+) \rightarrow \text { autophagy }(+) \rightarrow \text { HSCs } \\
\text { apoptosis }(+)\end{array}$ & {$[31]$} \\
\hline Dihydroartemisinin (DHA) & & Inducing HSCs senescence & $\begin{array}{l}\text { autophagy }(+) \rightarrow \text { GATA6 accumula- } \\
\text { tion }(+) \rightarrow \text { JNK1/2(+) } \rightarrow \text { p53 and } \\
\text { p16(+) } \rightarrow \text { aHSCs aging }(+)\end{array}$ & {$[36]$} \\
\hline Oroxylin A & & Inhibiting HSCs viability & $\begin{array}{c}\text { Atg3、Atg5 } 、 \text { Beclin1/Atg6 } 、 \operatorname{Atg} 12 \\
、 \operatorname{Atg} 14(+) \rightarrow \text { autophagy(+) }\end{array}$ & {$[37]$} \\
\hline Caffeic acid phenethyl ester & & & $\operatorname{AKT} / \mathrm{mTOR}(-) \rightarrow$ autophagy $(+)$ & {$[38]$} \\
\hline Betulinic acid & & & MAPK/ERK $(+) \rightarrow$ autophagy $(+)$ & [39] \\
\hline Dihydrotanshinone I (DH I) & & & $\begin{array}{l}\text { Hippo(-) } \rightarrow \text { YAP/ } \\
\text { TEAD2(-) } \rightarrow \text { autophagy(+) }\end{array}$ & {$[40]$} \\
\hline
\end{tabular}

Description: “+” means up-regulation or activation; “-” indicates decrease or inhibition; ” $\rightarrow$ means regulation/mediation 
Table 2 Molecular formulas and chemical structures of some natural compounds

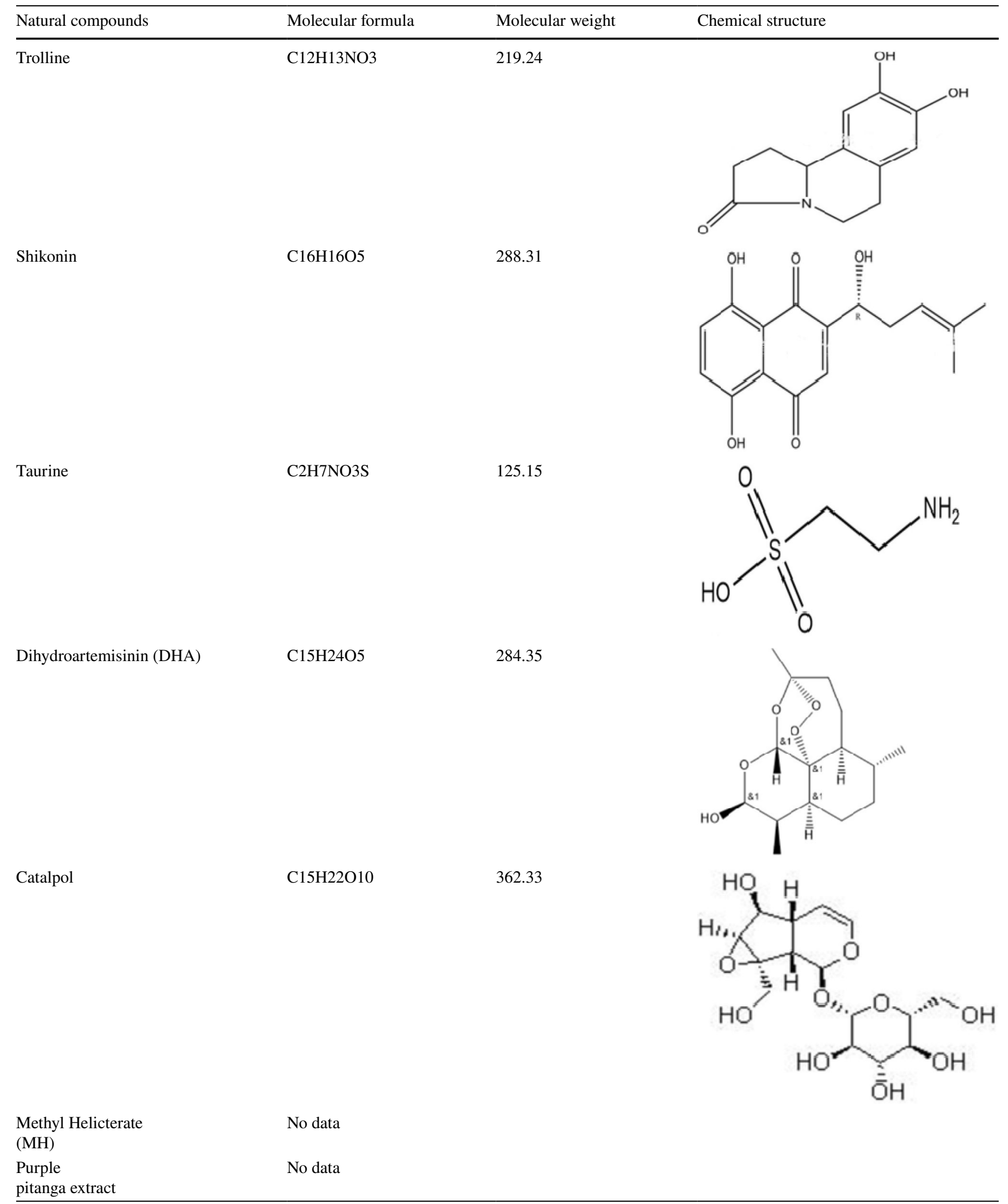


Table 2 (continued)

Natural compounds

Caffeine

Betulinic acid

$\mathrm{C} 30 \mathrm{H} 48 \mathrm{O} 3$

Oroxylin A

C16H12O5

Caffeic

acid

phenethyl ester(CAPE)

C18H14O3
Molecular formula

C8H10N4O2

194.19<smiles>CC1C(=O)c2c(ncn2C)C(C)C1=O</smiles><smiles>Cc1cccc2c3c(ccc12)C1=C(C(=O)C3=O)C(C)CO1</smiles>

456.70

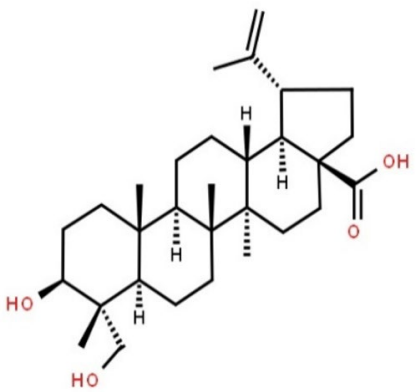

284.26<smiles>COc1c(O)cc2oc(-c3ccccc3)cc(=O)c2c1O</smiles>

284.31<smiles>O=C(OCCc1ccccc1)c1ccc(O)c(O)c1</smiles>

of autophagy. Since the TGF- $\beta 1 /$ Smads signaling pathway initiates transcription of the Beclin-1 gene to regulate autophagy, they further checked TGF- $\beta 1$ and phosphorylated Smad2/3 in both models and found both of them rising. Shikonin could downregulate their level in a dose-dependent manner. Therefore, they hypothesized that the mechanism of autophagy inhibition induced by shikonin may be partly due to the TGF- $\beta 1 /$ Smads signaling pathway suppression.

Taurine is a sulfur-containing amino acid existing in a variety of human and animal tissue, which can reduce liver damage caused by several toxic substances such as alcohol, heavy metals, and chemicals [21]. Wang [22] et al. found 
As $2 \mathrm{O} 3$ could stimulate the activation of LX-2 cells (a kind of immortalized and activated human HSC cell line) and the autophagy flux of them. Interestingly, the LX-2 cells activation was autophagy dependent, and taurine, though, could significantly relieve these effects. Previous studies indicated that the PPAR $\alpha$ could regulate autophagy. Their further study demonstrated the autophagy flux was decreased by inhibiting PPAR $\alpha$. Therefore, they speculated taurine may have a therapeutic effect on LF by inhibiting HSCs autophagy, which was regulated by PPAR $\alpha$.

\section{Natural compounds that enhance autophagy in HSCs to alleviate LF}

\section{Natural compounds that inhibit inflammation by boosting autophagy in HSCs}

Inflammation is involved in the whole process of LF. Inhibiting the release of inflammatory cytokines and improving the inflammatory microenvironment can even make the aHSCs convert to an inactivated state. Autophagy plays an important role in regulating the immune response and controlling inflammation. Specifically, autophagy controls inflammation through both directly inhibiting inflammatory complexes formation and indirectly suppressing it by removing damaged organelles or pathogenic microorganisms from cells. Thus, decreasing inflammatory response via regulating autophagy may have positive effects on LF.

It was reported that dihydroartemisinin (DHA), an effective antimalarial drug, could improve liver histopathology injuries and relieve LF in rat model [23]. Zhang [24] et al. found that DHA improved LF by restraining inflammatory responses. Meanwhile, autophagy level was upregulated in aHSCs. After down-regulating the autophagy of aHSCs with Atg5 siRNA, they found that DHA-induced antiinflammatory effect was removed, while transfection of Atg5 plasmids could enhance the anti-inflammatory effect. Therefore, autophagy was considered indispensable for the anti-inflammatory effect of DHA in LF. Their further study

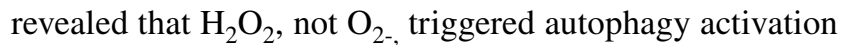
of aHSCs. The anti-inflammatory effect of DHA is associated with autophagy activation via enhancing ROS-JNK1/2 signal pathway.

Catalpol, an iridoid glycoside extracted from traditional Chinese medicine Rehmannia glutinosa, has remarkable pharmacological effects such as antioxidant, anti-inflammatory, anti-diabetic, anti-tumor [25]. Liu [26] et al. found that catalpol could inhibit the secretion of fiber ( $\alpha$-SMA, fibronectin and $\alpha-1$ procollagen) and inflammatory factors (IL-6, IL-8, TNF- $\alpha$ ) and up-regulate the level of autophagy in aHSCs. However, inhibiting autophagy dramatically reduced the effect of catalpol on the inhibition of inflammatory factors release and collagen secretion from aHSCs.
As a result, the function of catalpol in reducing LF through inflammation inhibition is autophagy dependent. Unfortunately, this study did not clarify the specific molecular mechanism between autophagy and inflammation regulation.

\section{Natural compounds that induce HSCs apoptosis by increasing autophagy}

Inducing aHSCs apoptosis is a strategy for the therapy of LF. A lot of natural compounds are cytotoxic to aHSCs. Contrary to well-known mechanisms of cell protection, autophagy also leads to cell death in specific cases, which is called autophagic cell death (ACD). ACD is the result of excessive cell autophagy, also being called II type programmed cell death. The main feature of ACD is that a large number of autophagy-lysosomes appear in cytoplasm, and most of the cytoplasmic material is degraded, but the nucleus remains integrity. Unlike apoptosis, ACD is generally independent of the activity of the caspase protein family [27]. Autophagy and apoptosis are two important cellular processes with complex and cross-protein networks. Bcl-2 has been identified as a central regulator of them. When nutrition is sufficient, Beclin-1 and Bax/BAK bind to Bcl-2 or Bcl-xL respectively, to prevent the initiation of autophagy and apoptosis. When under stress conditions, several mechanisms mediate the interruption of this interaction, thereby allowing them to be induced. In general, autophagy inhibits apoptosis by phagocytosis of pro-apoptotic caspases (e.g., caspase 8) and destruction of reactive mitochondria to prevent the release of cytochrome c. However, the autophagosome membrane serves as a platform for caspase- 8 activation and promotion of apoptosis mediated by the intracellular death-inducing signaling complex. Thus, Over-intense autophagy can also trigger the occurrence of apoptosis [28]. Some natural compounds can induce apoptosis of aHSCs by inhibiting autophagy in the early stages of their activation, while others promote aHSCs ACD or apoptosis which depends on intense and sustained autophagy.

Helicteres angustifolia is a widely used Chinese herbal medicine for immune diseases and liver diseases treatment. Zhang [29] et al. identified methyl helicterate (MH) as the active component of this herbal medicine. They found that MH could induce apoptosis of HSC-T6 cells and significantly up-regulate autophagy level. After inhibiting autophagy, MH lost this ability. In contrast, enhancing autophagy promoted this effect. To clarify whether MHinduced autophagy is associated with apoptosis, they examined two important signaling pathways, JNK and mTOR, involved in hepatic fibrosis formation and HSCs activation. They found that MH remarkably induced the activation of JNK signaling pathway, while, the PI3K/Akt/mTOR pathway was inhibited, which resulted in autophagy activation and apoptosis of HSCs. Therefore, enhancing autophagy to 
induce aHSCs apoptosis is the essence of MH's anti-hepatic fibrosis effect.

Eugenia uniflora, whose common name is pitanga or Brazilian cherry, is a plant widely distributed in South America. Its fruit has been proved to work as an antioxidant by inhibiting lipid peroxidation and scavenging free radicals. Denardin [30] et al. found that purple pitanga extract can reduce the viability of GRX cells (a kind of aHSCs line). They also found it could significantly induce autophagy and mitochondrial autophagy in GRX cells. However, enhanced autophagy not only impaired mitochondrial function, but declined mitochondrial content in the GXR cells, ultimately promoting GXR cells apoptosis via mitochondria-dependent pathways, which suggested that autophagy/mitochondrial autophagy induced by purple pitanga extract did not play a cytoprotective role, in contrast, leading to cell death. However, the molecular mechanism of the link between autophagy and apoptosis induced by purple pitanga extract remains unclear.

The protective effect of coffee and caffeine on the liver is drawing public attention. A number of studies have reported that regular intake of a certain amount of coffee can slow down the progression of chronic liver diseases. Li [31] et al. chose LX-2 cells as the research object and found that caffeine inhibited the vitality of LX-2 cells and induced apoptosis. They observed that caffeine induced endoplasmic reticulum (ER) stress and autophagy. Apoptosis of LX-2 cells was effectively weakened when autophagy was inhibited. After knocking down the IRE1- $\alpha$ gene, which regulates ER stress, the autophagic flux of LX-2 cells decreased. Based on the results, they believed that caffeine induces aHSCs apoptosis through autophagy, mediated by ER stress. However, this effect needs to be further verified in more HSCs lines, and the specific mechanism of autophagy leading to apoptosis is also worth further study.

\section{Natural compounds that induce HSCs senescence by increasing autophagy}

Previous studies also indicated a close relationship between autophagy and cellular senescence [32,33], and aging HSCs have been found in liver fibrosis tissue [34]. Krizhanovsky [35] et al. reported that aging aHSCs secreted less ECM and promoted the reversal of LF. Thus, inducing aHSCs senescence may be a strategy to relieve LF.

Zhang [36] et al. found DHA-induced aHSCs senescence and attenuated LF in rats, and further confirmed the transcription factor GATA6 was the upstream signaling molecule for this effect. Indeed, their study showed that the accumulation of GATA6 could lead to the number of aging aHSCs rising. Besides, DHA could also induce autophagy and there was a close tie between the level of autophagy and GATA6. When autophagy was inhibited, the amount of GATA6 declining which led to the loss of senescent HSCs. Therefore, the HSCs aging induced by DHA is autophagy-dependent.

\section{Natural compounds that inhibit HSCs viability by increasing autophagy}

As mentioned above, most studies have shown that inhibition of autophagy can reduce the activity of aHSCs, and a lot of drugs exert anti-fibrosis effects through this mechanism. However, some drugs are found to inhibit HSCs activity by promoting autophagy and these drugs do not significantly increase the number of aging or apoptosis aHSCs, but inhibit their activity. Some of them work by enhancing autophagy to interfere with signaling pathways closely related to HSCs activation. Disappointedly, the mechanism of some other drugs remains unclear.

Oroxylin A is a natural mono-flavonoid in Scutellaria, a kind of Chinese herb, which has been proved to have anti-inflammatory, anti-angiogenic, antioxidative, and antitumor pharmacological activities. Chen [37] et al. found that Oroxylin A could decrease the deposition of ECM in CCl4induced LF mice and down-regulated the expression of COL-1, $\alpha$-SMA, PDGF- $\beta$ R and TGF- $\beta$ R which were all the molecular markers of aHSCs. Also, Oroxylin A upregulated the autophagy level in aHSCs remarkably. The anti-fibrotic effect of Oroxylin A completely lost after using autophagy inhibitor 3-MA, suggesting that this anti-fibrotic effect of Oroxylin A was triggered by activating autophagy. However, this study did not further explore the molecular mechanism of autophagy to reduce the activity of HSCs.

Caffeic acid phenethyl ester (CAPE) is one of the main medicinal components of propolis. CAPE is reported to have a variety of biological activities, such as anti-inflammatory, antioxidant, anti-tumor. Yang [38] et al. found that CAPE effectively attenuated CCl4-induced LF and inhibited the activity of HSCs. They observed the autophagy was upregulated in CAPE-treated HSC-T6 cells. To verify the role of autophagy, they used rapamycin (a kind of autophagy agonist) and 3-MA to treat HSC-T6 cells respectively with CAPE. The results showed that the rapamycin group further promoted autophagy and inhibited HSC-T6 cells activity, whereas 3-MA went the opposite direction. Meanwhile, they also confirmed that CAPE may attenuate liver fibrosis by inhibiting AKT/mTOR signaling pathway.

Betulinic acid (BA) is a natural triterpenoid, which is reported to have liver protective effect. Liu [39] et al. found that BA reduced the serum level of platelet-derived growth factor (PDGF) and hydroxyproline (HYP) in LF mice and the expression of $\alpha$-SMA and collagen I. They also found that BA enhanced autophagy in both vivo and vitro. To verify whether this effect of BA was associated with autophagy, their further study showed that the effect mentioned above 
by BA was significantly relieved after using autophagy inhibitors, conveying that autophagy played a key role. Surprisingly, they validated that the role of BA in promoting autophagy was realized through inhibiting MAPK/ERK signaling pathway.

Dihydrotanshinone I (DHI), a lipophilic component extracted from Salvia miltiorrhiza Bunge (Tanshen), has liver protection, anti-inflammatory, and weight-loss effects reported in previous studies. Ge [40] et al. found that DHI strongly repressed hepatic fibrogenic genes expression and in BDL-induced LF rats and LX-2 cells. In their study, DHI was also found to have a strong inhibitory effect on YAP/TEAD2 complex formation, the downstream molecular event of Hippo pathway which was closely associated with HSC activation. Simultaneously, they discovered that the autophagy flux was much higher in DHI treated HSCs. Based on these facts, they speculated that there might be some connection between autophagy and Hippo pathway. After YAP was knocked out, autophagy level in DHI treatment group was further increased and the anti-fibrotic effect was enhanced. Nevertheless, overexpression of YAP yielded the opposite results. In summary, DHI could alleviate LF by preventing the YAP/TEAD2 complex formation and stimulate autophagy. However, the underlying mechanism of YAP and autophagy requires further exploration.

\section{Conclusions and future prospective}

Natural compounds have different effects on HSCs by regulating autophagy. Current studies indicate that the role of autophagy in LF is complicated. The effects of autophagy on HSCs may depend on its insensity and duration. A degree of autophagy enhancement helps activate quiet HSCs and maintain their activated phenotypes later on. While, in aHSCs, autophagy overexpression may lead aHSCs to be senescent, autophagic cell death or apoptosis [41]. As mentioned above, either over-strong or over-weak autophagy is a threat to the survival of cells. Therefore, enhancing or weakening autophagy both may play a role in promoting the inactivation or death of HSCs. The pharmacological effects of natural compounds are complex and may interfere with various life activities in cells. Therefore, to identify whether autophagy plays a major role and the underlying molecular mechanisms seems indispensable. Besides, the effects of changed autophagy level on other liver cells should not be ignored, because these non-stellate cells are also involved in the formation of LF, and the effect of autophagy on them is even opposite to that of HSC. For example, chronic liver cell damage is another key step in the development of LF, and autophagy has a protective effect on liver cells [42]. So, drugs targeting autophagy should be the focus of future research. Finally, a lot of anti-liver fibrosis drugs have poor clinical curative effect, possibly because basic research only focuses on the cellular or molecular level, which does help clarify pharmacological mechanisms but omits the complexity of biological systems [43]. Hence, it is of great significance to explore the metabolic differences between humans, animals, and cells.

Conclusively, natural compounds have a lot of advantages such as abundant reserves, wide sources, and relative safety. Developing natural drugs with anti-fibrosis effects has broad prospects and great significance.

Acknowledgments Thanks for the suggestion on this article from Professor Jianchang Shu and Doctor Xuyou Liu.

Funding The study was funded by the Scientific Research Project of Guangzhou Science Technology and Innovation Commission. [grant number: No.201707010356].

\section{Compliance with ethical standards}

Conflict of Interest Author Yongxiang Shu declares that he has no conflict of interest. Author Xuyou Liu declares that he has no conflict of interest. Author Haifeng Huang declares that he has no conflict of interest. Author Qi Wen declares that she has no conflict of interest. Author Jianchang Shu declares that he has no conflict of interest.

Ethical approval This article does not contain any studies with human participants or animals performed by any of the authors.

Open Access This article is licensed under a Creative Commons Attribution 4.0 International License, which permits use, sharing, adaptation, distribution and reproduction in any medium or format, as long as you give appropriate credit to the original author(s) and the source, provide a link to the Creative Commons licence, and indicate if changes were made. The images or other third party material in this article are included in the article's Creative Commons licence, unless indicated otherwise in a credit line to the material. If material is not included in the article's Creative Commons licence and your intended use is not permitted by statutory regulation or exceeds the permitted use, you will need to obtain permission directly from the copyright holder. To view a copy of this licence, visit http://creativecommons.org/licenses/by/4.0/.

\section{References}

1. Parola M, Pinzani M (2019) Liver fibrosis: pathophysiology, pathogenetic targets and clinical issues. Mol Asp Med 65:37-55. https://doi.org/10.1016/j.mam.2018.09.002

2. Farrell GC, Larter CZ (2006) Nonalcoholic fatty liver diseases: from steatosis to cirrhosis. Hepatology 43:S99-s112. https://doi. org/10.1002/hep.20973

3. Friedman SL, Bansal MB (2006) Reversal of hepatic fibrosis fact or fantasy? Hepatology 43:S82-S88. https://doi.org/10.1002/ hep. 20974

4. Tanida I, Ueno T, Kominami E (2008) LC3 and Autophagy. Methods Mol Biol 445:77-88. https://doi.org/10.1007/978-1-59745 -157-4_4

5. Yu Z, Ma J, Li X et al (2018) Autophagy defects and related genetic variations in renal cell carcinoma with eosinophilic 
cytoplasmic inclusions. Sci Rep 8:9972. https://doi.org/10.1038/ s41598-018-28369-y

6. Friedman SL (1998) Cellular networks in hepatic fibrosis. Digestion 59(4):368-371. https://doi.org/10.1159/000007517

7. Higashi T, Friedman SL, Hoshida Y (2017) Hepatic stellate cells as key target in liver fibrosis. Adv Drug Deliv Rev 121:27-42. https://doi.org/10.1016/j.addr.2017.05.007

8. Friedman SL (2015) Hepatic fibrosis: emerging therapies. Dig Dis 33(4):504-507. https://doi.org/10.1159/000374098

9. Czaja MJ (2011) Functions of autophagy in hepatic and pancreatic physiology and diseases. Gastroenterology 140:1895-1908. https ://doi.org/10.1053/j.gastro.2011.04.038

10. Ke PY (2019) Diverse functions of autophagy in liver physiology and liver diseases. Int J Mol Sci pii E300. https://doi.org/10.3390/ ijms 20020300

11. Trautwein C, Friedman SL, Schuppan D et al (2015) Hepatic fibrosis: concept to treatment. J Hepatol 62:S15-S24. https://doi. org/10.1016/j.jhep.2015.02.039

12. Zhang Z, Zhao S, Yao Z et al (2017) Autophagy regulates turnover of lipid droplets via ROS-dependent Rab25 activation in hepatic stellate cell. Redox Biol 11:322-334. https://doi.org/10.1016/j.redox .2016.12.021

13. Zhang XY, Shu JC, Huang ML et al (2019) Curcumin combined with metformin inhibits autophagy and promotes apoptosis in LX-2 hepatic stellate cells. China Modern Med J 21(4):1-4. https://doi. org/10.3969/j.issn.1672-9463.2019.04.001

14. Song HD, Pan J, Ouyang P et al (2018) Effect of curcumin on the expression of autophagy- related proteins in rats with liver fibrosis. New Med 49(8):579-582. https://doi.org/10.3969/j. issn.0253-9802.2018.08.009

15. Seo HY, Jang BK, Jung YA et al (2014) Phospholipase D1 decreases type I collagen levels in hepatic stellate cells via induction of autophagy. Biochem Biophys Res Commun 449:38-43. https://doi. org/10.1016/j.bbrc.2014.04.149

16. Liu XY (2015) Induction of autophagy and apoptosis by miR-148a through the sonic hedgehog signaling pathway in hepatic stellate cells. Am J Cancer Res 5(9):2569-2589

17. Ni T, He Z, Dai Y et al (2017) Oroxylin a suppresses the development and growth of colorectal cancer through reprogram of HIF1alpha-modulated fatty acid metabolism. Cell Death Dis 8:e2865. https ://doi.org/10.1038/cddis.2017.261

18. Bai F, Huang Q, Nie J et al (2017) Trolline ameliorates liver fibrosis by inhibiting the NF-kappaB pathway, promoting HSC apoptosis and suppressing autophagy. Cell Physiol Biochem 44:436-446. https ://doi.org/10.1159/000485009

19. Liu T, Xia Y, Li J et al (2016) Shikonin attenuates concanavalin A-induced acute liver injury in mice via inhibition of the JNK pathway. Mediat Inflamm 2016:2748367. https://doi. org/10.1155/2016/2748367

20. Liu T, Xu L, Wang C et al (2019) Alleviation of hepatic fibrosis and autophagy via inhibition of transforming growth factor-beta1/Smads pathway through shikonin. J Gastroenterol Hepatol 34:263-276. https://doi.org/10.1111/jgh.14299

21. Murakami S, Ono A, Kawasaki A et al (2018) Taurine attenuates the development of hepatic steatosis through the inhibition of oxidative stress in a model of nonalcoholic fatty liver diseases in vivo and in vitro. Amino Acids 50:1279-1288. https://doi.org/10.1007/s0072 6-018-2605-8

22. Wang Z, Tao Y, Qiu T et al (2019) Taurine protected As2O3-induced the activation of hepatic stellate cells through inhibiting PPARalphaautophagy pathway. Chem Biol Interact 300:123-130. https://doi. org/10.1016/j.cbi.2019.01.019

23. Tai $X$, Cai $X B$, Zhang $Z$ et al (2016) In vitro and in vivo inhibition of tumor cell viability by combined dihydroartemisinin and doxorubicin treatment, and the underlying mechanism. Oncol Lett 12:3701-3706. https://doi.org/10.3892/ol.2016.5187
24. Zhang Z, Guo M, Zhao S et al (2016) ROS-JNK1/2-dependent activation of autophagy is required for the induction of antiinflammatory effect of dihydroartemisinin in liver fibrosis. Free Radic Biol Med 101:272-283. https://doi.org/10.1016/j.freeradbio med.2016.10.498

25. Zhu P, Wu Y, Yang A et al (2017) Catalpol suppressed proliferation, growth and invasion of CT26 colon cancer by inhibiting inflammation and tumor angiogenesis. Biomed Pharmacother 95:68-76. https ://doi.org/10.1016/j.biopha.2017.08.049

26. Liu Z, Zhu P, Zhang L et al (2018) Autophagy inhibition attenuates the induction of anti-inflammatory effect of catalpol in liver fibrosis. Biomed Pharmacother 103:1262-1271. https://doi.org/10.1016/j. biopha.2018.04.156

27. Wu SL, Huang YY, Ding HL et al (2017) Progress in the study of autophagy, autophagic cell death and tumor. Life Sci 29(8):763-768. https://doi.org/10.13376/j.cbls/2017103

28. Mukhopadhyay S, Panda PK, Sinha N et al (2014) Autophagy and apoptosis: where do they meet? Apoptosis 19(4):555-566. https:// doi.org/10.1007/s10495-014-0967-2

29. Zhang XL, Chen ZN, Huang QF et al (2018) Methyl Helicterate inhibits hepatic stellate cell activation through modulation of apoptosis and autophagy. Cell Physiol Biochem 51:897-908. https://doi. org/10.1159/000495390

30. Denardin CC, Martins LA, Parisi MM et al (2017) Autophagy induced by purple pitanga (Eugenia uniflora L.) extract triggered a cooperative effect on inducing the hepatic stellate cell death. Cell Biol Toxicol 33:197-206. https://doi.org/10.1007/s1056 5-016-9366-5

31. Li YJ (2017) Caffeine induces hepatic stellate cell apoptosis by enhancing endoplasmic reticulum stress-mediated autophagy. Dissertation, Shanghai Jiaotong University

32. Zhang H, Puleston DJ, Simon AK (2016) Autophagy and immune senescence. Trends Mol Med 22:671-686. https://doi.org/10.1016/j. molmed.2016.06.001

33. Kang C, Elledge SJ (2016) How autophagy both activates and inhibits cellular senescence. Autophagy 12:898-899. https://doi. org/10.1080/15548627.2015.1121361

34. Jin H, Lian N, Zhang F et al (2016) Activation of PPARgamma/ P53 signaling is required for curcumin to induce hepatic stellate cell senescence. Cell Death Dis 7:e2189. https://doi.org/10.1038/cddis .2016 .92

35. Krizhanovsky V, Yon M, Dickins RA et al (2008) Senescence of activated stellate cells limits liver fibrosis. Cell 134:657-667. https ://doi.org/10.1016/j.cell.2008.06.049

36. Zhang Z, Yao Z, Zhao S et al (2017) Interaction between autophagy and senescence is required for dihydroartemisinin to alleviate liver fibrosis. Cell Death Dis 8:e2886. https://doi.org/10.1038/cddis .2017 .255

37. Chen W, Zhang Z, Yao Z et al (2018) Activation of autophagy is required for Oroxylin a to alleviate carbon tetrachloride-induced liver fibrosis and hepatic stellate cell activation. Int Immunopharmacol 56:148-155. https://doi.org/10.1016/j.intimp.2018.01.029

38. Yang N, Dang S, Shi J et al (2017) Caffeic acid phenethyl ester attenuates liver fibrosis via inhibition of TGF-beta1/Smad3 pathway and induction of autophagy pathway. Biochem Biophys Res Commun 486:22-28. https://doi.org/10.1016/j.bbrc.2017.02.057

39. Liu Y, Bi Y, Mo C et al (2019) Betulinic acid attenuates liver fibrosis by inducing autophagy via the mitogen-activated protein kinase/ extracellular signal-regulated kinase pathway. J Nat Med 73:179_ 189. https://doi.org/10.1007/s11418-018-1262-2

40. Ge M, Liu H, Zhang Y et al (2017) The anti-hepatic fibrosis effects of dihydrotanshinone I are mediated by disrupting the yes-associated protein and transcriptional enhancer factor D2 complex and stimulating autophagy. Br J Pharmacol 174:1147-1160. https://doi. org/10.1111/bph.13766 
41. Wang W, Yan J, Wang H et al (2014) Rapamycin ameliorates inflammation and fibrosis in the early phase of cirrhotic portal hypertension in rats through inhibition of mTORC1 but not mTORC2. PLoS One 9:e83908. https://doi.org/10.1371/journal.pone.0083908

42. Song Y, Zhao Y, Wang F et al (2014) Autophagy in hepatic fibrosis. Biomed Res Int 2014:436242

43. Ezhilarasan D, Sokal E, Najimi M (2018) Hepatic fibrosis: it is time to go with hepatic stellate cell-specific therapeutic targets. Hepatobiliary Pancreat Dis Int 17:192-197. https://doi. org/10.1016/j.hbpd.2018.04.003

Publisher's Note Springer Nature remains neutral with regard to jurisdictional claims in published maps and institutional affiliations. 